\title{
Efecto Bioprotector de Micorrizas Arbusculares en la Reducción de Roya (Hemileia vastatrix) en la Región San Martín
}

\author{
Bioprotective Effect of Arbuscular Mycorrhizae in the \\ Reduction of Rust (Hemileia vastatrix) in the San Martín \\ Region \\ Vallejos-Torres, Geomar ${ }^{1}$ [0000-0001-7084-977X], Saboya, Anabel ${ }^{2}$ [0000-0003- \\ 0105-3764] y Arévalo, Luis ${ }^{2}$ [0000-0002-6417-8161] \\ ${ }^{1}$ Universidad Nacional de San Martín, Tarapoto, Perú \\ ${ }^{2}$ Instituto de Investigaciones de la Amazonía Peruana, Perú \\ gvallejostegmail.com
}

Citar como: Vallejos Torres, G., Saboya Pisco, A., \& Arevalo Lopez, L. A. (2021). Efecto Bioprotector de Micorrizas Arbusculares en la Reducción de Roya (Hemileia vastatrix) en la Región San

Martín. Revista

Agrotecnológica

Amazónica, 1(1), 34-44. https://doi.org/10.51252/ra a.v1i1.122

Recibido: $15 / 11 / 2020$

Revisado: $15 / 12 / 2020$

Publicado: 31/01/2021
Resumen. La roya es un problema generalizado que ha afectado a diferentes países de América Latina, generando pérdidas económicas en los productores cafetaleros. Se evaluó el efecto de los hongos micorrízicos arbusculares (HMA), sobre la presencia de roya (Hemileia vastatrix) en plantas de café. Se empleó un diseño completamente al azar con dos factores: fuentes de inóculo HMA e Inoculación de roya, incluyendo tratamiento testigos (absoluto e infestado); se usó como sustrato tierra agrícola y arena (2:1); se inoculó plantas de café con 1500 esporas de HMA procedente de Rioja, Lamas, Dorado y Huallaga, posteriormente se inóculo con esporas de roya; se evaluaron altura de planta, área foliar, biomasa seca y aérea, incidencia, severidad, colonización micorrízica y longitud de micelio extraradical. Los resultados mostraron que los tratamientos inoculados con HMA presentaron mejoras incrementos en morfología, micorrización y menor incidencia y severidad a roya.

Palabras clave: hongos micorrízicos arbusculares, roya, plantas de café

Abstract. Rust is a widespread problem that has affected different countries in Latin America, generating economic losses in coffee producers. The effect of arbuscular mycorrhizal fungi (AMF) on the presence of rust (Hemileia vasta-trix) in coffee plants was evaluated. A Completely Random Design was used with two factors: AMF inoculum sources and rust inoculation, including control treatments (absolute and infested); agricultural land and sand were used as substrate (2: 1); Coffee plants were inoculated with 1500 AMF spores from Rioja, Lamas, Dorado and Huallaga, later it was inoculated with rust spores; Plant height, leaf area, dry and aerial biomass, incidence, severity, mycorrhizal colonization and extraradical mycelium length were evaluated. The results showed that the treatments inoculated with AMF presented improvements, increases in morphology, mycorrhization and lower incidence and severity of rust.

Keywords: Arbuscular mycorrhizal fungi, coffee plants, rust 


\section{Introducción}

El Café (Coffea arabica L.), es el principal producto agrícola de exportación del Perú, con gran importancia económica y social (Palomino et al. 2014), entre las cuales se encuentra la Región San Martín que en el año 2015 este cultivo representó el 14,7 \% de la producción nacional, ocupando el cuarto puesto con 540 mil toneladas; siendo por ello el eje económico de miles de familias contribuyendo significativamente al PBI. En el mes abril de 2020 el precio promedio FOB fue de USD 1298 t. lo cual significó una variación de -44,5 \% en comparación al mismo periodo del año pasado.

En lo que va del año se ha exportado 15824 toneladas de este producto, y las exportaciones de café disminuyeron en 46,0 \%, sin embargo, el precio promedio presentó un incremento de 8,2 \% en comparación al mismo periodo del año anterior (MINAGRI, 2020). La producción del café ha tenido altos y bajos durante la historia, siendo uno de los problemas principales que merma la producción la enfermedad de roya, cuyo agente causante es el hongo Hemileia vastatrix, es destructiva a nivel mundial con gran impacto económico y social (Dalmazzone, 2002).

Una de las innovaciones respecto a este cultivo es la inoculación con micorrizas, la cual está sustentada en brindar mayor fortaleza al sistema radicular de la planta que lo vuelve resistente ante el ataque de cualquier patógeno (Sevilla, 2004). Reportes científicos indican que la patogenicidad a roya disminuye a mayor longitud de micelio y colonización micorrízica (Herrera et al. 2019).

En el presente estudio se tuvo como objetivo evaluar y analizar los efectos de bioprotección en el cultivo del café variedad Caturra, con aplicación de micorrizas arbusculares ya que estos microorganismos mejoran el sistema radicular del café, mejora las funciones fisiológicas y transporte del agua y los minerales del suelo, el cual disminuye el estrés hídrico, estimula el crecimiento de las células epidérmicas haciendo menos vulnerables a patógenos del suelo y como consecuencia la enfermedad de la roya en café variedad caturra se puede ver favorecido o desfavorecido en la incidencia y severidad.

\section{Materiales y Métodos}

\section{Ubicación y caracterización del área de estudio}

El trabajo de investigación se desarrolló en la localidad de (Alto Chontal), ubicado en el distrito y provincia de Lamas y región San Martín. Fue establecido bajo condiciones de vivero expuestas al campo.

\section{Inoculación con Hongos micorrízicos arbusculares}

Citar como: Vallejos Torres, G., Saboya Pisco, A., \& Arevalo Lopez, L. A. (2021). Efecto Bioprotector de Micorrizas Arbusculares en la Reducción de Roya (Hemileia vastatrix) en la Región San Martín. Revista Agrotecnológica Amazónica, 1(1), 34-44. https://doi.org/10.51252/raa.v1i1.122 
La inoculación tuvo su inicio en la multiplicación y conteo de esporas de HMA por cada inóculo procedente de las provincias de Rioja (Oriente Nuevo), Lamas (Chirapa), El Dorado (Buena Vista) y Huallaga (Nuevo Brazil). Se aplicó 1500 esporas de HMA por plántula de café de la variedad caturra propagada en sustrato tierra agrícola y arena esterilizada en autoclave a $121^{\circ} \mathrm{C}$ por 1 hora, a $15 \mathrm{lb}$.

\section{Cuantificación y aplicación de las urediniosporas de roya a las plántulas de café.}

La cuantificación de la concentración de urediniosporas de roya permitió determinar el número de unidades infectivas. Con una pipeta se tomó una alícuota de la última dilución y se llenó la cámara de Neubauer por capilaridad, luego se llevó al microscopio y se procedió a contar los conidios en el cuadrante central de la cámara, en la que se consideró cinco cuadrados, los 4 de las esquinas y el del centro. Con esto se determinó las conidias por ml. mediante la siguiente fórmula, descrita por French y Hebert (1982) (Anexo 1).

Número de conidias/g= número de conidias $\mathrm{x}$ factor cámara $\mathrm{x}$ factor dilución.

\section{Evaluación de la micorrización y variable smorfológico.}

La colonización micorrízica se dio inicio con la tinción de raíces de café, proceso desarrollado de acuerdo a la metodología de Viertheilig et al. 1998 modificado. Se observó cada campo visual, la intersección de las raíces con la línea horizontal, anotando la presencia o ausencia de colonización micorrízica del segmento de la raíz que está en contacto con la línea de la cuadricula. Para la longitud de micelio se realizó utilizando la técnica del gel semisólido y cuantificación por el método de intersección de cuadrantes (Robles, 2009).

Se evaluó el área foliar, severidad a roya en plantas de café, longitud de micelio extraradical (MER) y colonización micorrízica.

\section{Diseño estadístico}

El diseño experimental en este trabajo de investigación se desarrolló utilizando el diseño completamente al azar (DCA), con arreglo factorial de 5A x 2B, considerando el factor A (fuentes de Inóculo (HMA) y el factor B (Inoculación de roya) con 3 repeticiones por tratamiento, haciendo 30 unidades experimentales, con un nivel de significancia de $p<0,05$ y 0,01 y probabilidad de error para determinar la confiabilidad y naturaleza de las diferencias entre tratamientos (Padrón, 1996). Todos los datos obtenidos se sometieron a un análisis de varianza, previo para el análisis de varianza se sometieron los datos a la evaluación del supuesto de normalidad utilizándose la dócima de Shapiro Wilk (Diz, 2008) y para el supuesto de homogeneidad de varianza se utilizó la dócima de Levene (Font, 2007), cumpliendo con los dos supuestos. Finalmente, los datos fueron sometidos a la prueba Tukey con un nivel de significancia de $\mathrm{p}<0,05$. Se almacenaron y analizaron los datos en el software SAS 9,2.

Citar como: Vallejos Torres, G., Saboya Pisco, A., \& Arevalo Lopez, L. A. (2021). Efecto Bioprotector de Micorrizas Arbusculares en la Reducción de Roya (Hemileia vastatrix) en la Región San Martín. Revista Agrotecnológica Amazónica, 1(1), 34-44. https://doi.org/10.51252/raa.v1i1.122 


\section{Resultados y discusiones}

\section{Área foliar en plantas de café.}
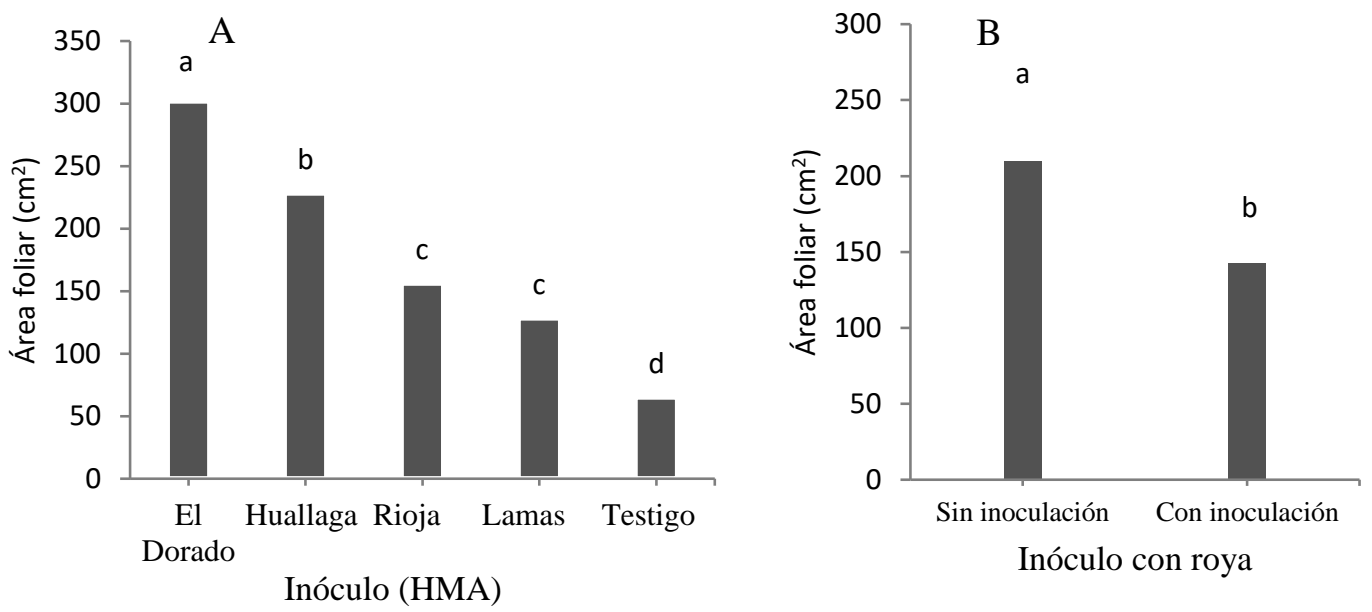

Figura 1. Prueba de Tukey $(\mathrm{p}<0,05)$ para el área foliar; A. Inoculación con HMA y B. Inoculación con roya. Medias con letras iguales no difieren estadísticamente entre sí

En el análisis de comparación de medias de Tukey $(\mathrm{p}<0,05)$ (figura 1A) para el efecto fuentes de inóculo (HMA) para el área foliar (cm2), se observó diferencias significativas entre las cuatro zonas. La fuente de inóculo El Dorado muestra los mejores resultados en área foliar, seguido por la fuente de inóculo de Huallaga, Rioja y Lamas. Comparados con los tratamientos testigos, quienes mostraron menor área foliar. Estos resultados demuestran que los HMA si influenciaron sobre el área foliar en las plantas de café, al mostrarse como mayor follaje al verse reflejado en plantas con un mayor volumen foliar. Semejante reportó Del águila (2016), los resultados obtenidos, muestran que la inoculación con HMA nativos, resultó provechosa, lográndose incrementos en el área foliar que fluctuaron entre 77 \% más con relación a las plantas no inoculadas. Asimismo, Fernández et al., (2005), en sus investigaciones realizadas en café en etapa de vivero, obtuvo incrementos entre $6 \%$ y $140 \%$ de área foliar con respecto a plantas no inoculadas con HMA, resultados similares a lo obtenido por Barrer (2009), donde tratamientos con HMA utilizados en su ensayo dieron un incremento en área foliar de 5 y $50 \%$ con respecto al testigo absoluto y de un 9 y $66 \%$ con respecto al testigo infestado con Meloidogyne spp. Así mismo semejante los resultados obtuvo Chinchay (2016), con respecto al efecto de los CHMA ya que obtuvo áreas mucho más elevadas comparando al testigo.

En el análisis de comparación de medias de Prueba de Tukey ( $\mathrm{p}<0.05)$ (figura 1B) para el efecto inoculación de roya para el área foliar (cm2), se observó diferencias significativas en las plantas inoculadas con roya y las no inoculadas, las plantas que no fueron inoculadas con roya muestran mejor área foliar a comparación de las que fueron inoculadas con esto se refuerza a lo explicado por (Auge, 2001), en la que manifiesta que la micorrización de hongo-planta, genera incrementos 
de las tasas fotosintéticas, logrando plantas con buenas características en estas dos variables, principalmente en la parte foliar.

\section{Incidencia de roya en plantas de café.}
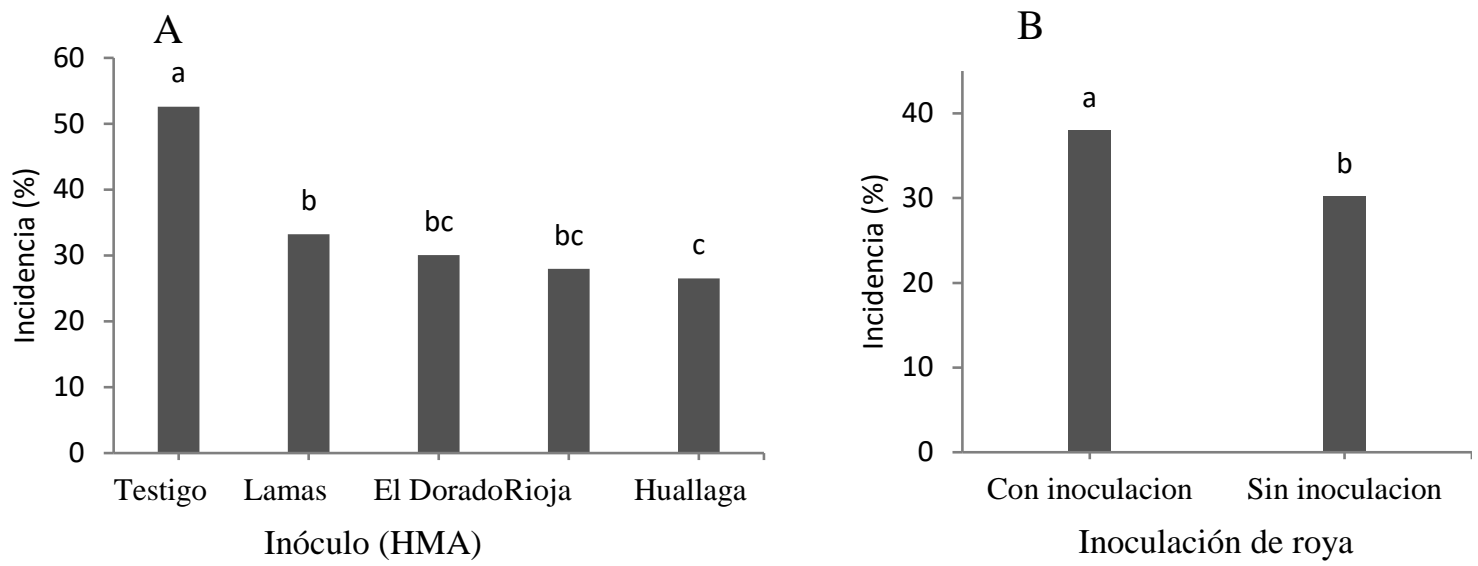

Figura 2. Prueba de Tukey $(\mathrm{p}<0,05)$ para la incidencia a roya; A. Inoculación con HMA y B. Inoculación con roya. Medias con letras iguales no difieren estadísticamente entre sí

El análisis de comparación de medias de Tukey $(\mathrm{p}<0,05)$ (figura $2 \mathrm{~A})$ muestra que el testigo, tuvo mayor incidencia de roya, con un porcentaje $52,61 \%$; comparados con las plantas que fueron inoculados con fuentes de HMA que presentan niveles inferiores en incidencia; para el factor independiente inoculación con roya el tratamiento en base a inoculación presento una mayor incidencia a roya (figura 2B). La infestación del testigo con roya; tres días antes que lleguen a campo definitivo (Alto Chontal - Lamas), explica que ha existido una inoculación natural a todos los tratamientos; a pesar de ello las plantas de café inoculadas con roya tuvieron menor infestación que los testigos esto quiere decir que los HMA al mejorar la calidad de vida de la plántula como la absorción de agua y fosforo que es importante a nivel de ácido nucleico para la formación de aminoácidos y proteínas estructurales y funcionales mejorando la calidad de vida de la planta dándoles mayor resistencia ante el ataque de hongos en este caso a Hemileia vastatrix, hongo causante de la roya del cafeto. La menor infección que se observa en las plantas inoculadas con HMA es debido a que la infección a nivel de campo fue más tardía y por consiguiente la incidencia es baja esto.

\section{Severidad de roya en plantas de café.}

Citar como: Vallejos Torres, G., Saboya Pisco, A., \& Arevalo Lopez, L. A. (2021). Efecto Bioprotector de Micorrizas Arbusculares en la Reducción de Roya (Hemileia vastatrix) en la Región San Martín. Revista Agrotecnológica Amazónica, 1(1), 34-44. https://doi.org/10.51252/raa.v1i1.122 

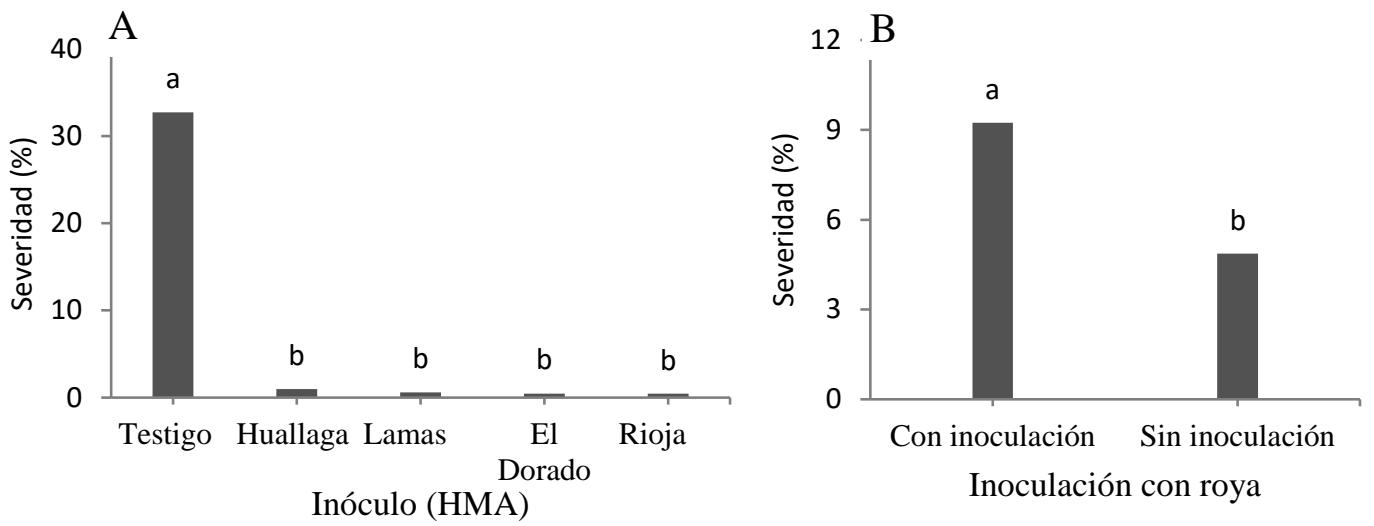

Figura 3. Prueba de Tukey $(\mathrm{p}<0,05)$ para la severidad a roya; A. Inoculación con HMA y B. Inoculación con roya. Medias con letras iguales no difieren estadísticamente entre sí

El análisis de comparación de medias de Tukey $(\mathrm{p}<0,05)$ (figura 3A) muestra mayor porcentaje de severidad el testigo sin la aplicación de micorrizas arbusculares (figura 3A); asimismo, se obtuvo mayor severidad a roya en plantas que fueron inoculadas con roya (figura 3B). Comparados las plantas que fueron inoculadas con las fuentes de inóculo de las cuatro provincias, quienes presentaron menor porcentaje de severidad ante el ataque. De acuerdo con Bock et al. (2010), el número de lesiones influye en la precisión y exactitud de las estimaciones visuales del área dañada; además, señala que, pero lo que refleja el gran grado de dispersión que existe entre las plantas que fueron inoculados con HMA y el testigo sin inoculación.

\section{Longitud de micelio extraradical (MER) y Porcentaje de colonización micorrízica.}
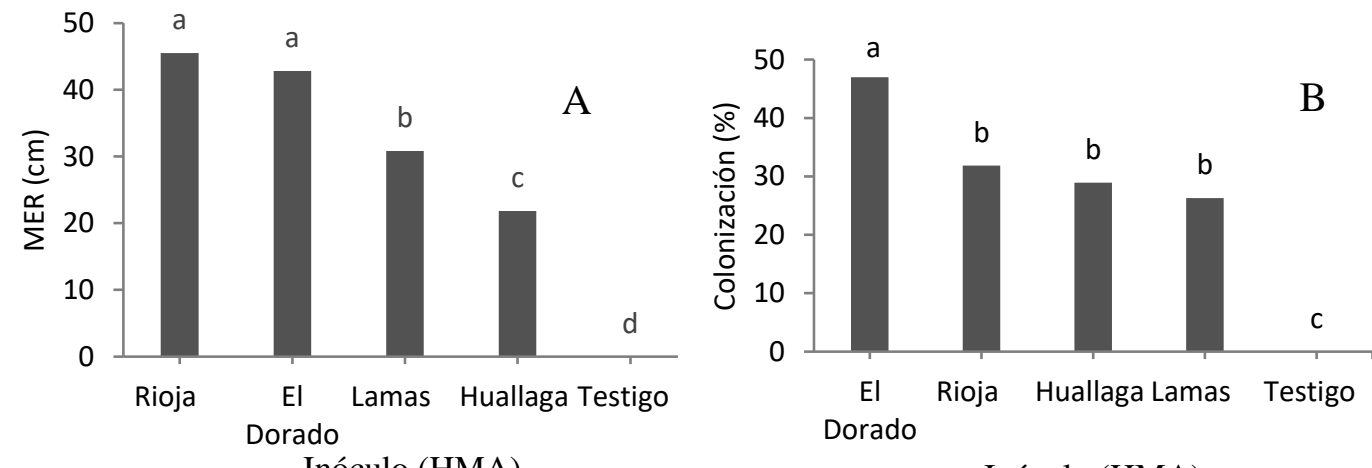

Fig. 4. Prueba de Tukey $(p<0,05)$ para micelio extraradicular y colonización micorrízica; A. Inoculación con HMA y B. Inoculación con roya. Medias con letras iguales no difieren estadísticamente entre sí

El análisis de comparación de medias de Tukey $(\mathrm{p}<0,05)$ (figura 4A) muestra que la fuente de inoculo de Rioja sobresalió en mayor longitud de micelio, seguido de la fuente de inóculo del El Dorado. Comparados con los tratamientos testigos, quienes mostraron resultados de $0 \mathrm{~cm}$ por no haber sido inoculados con esporas de HMA respectivamente. Asimismo, los inóculos procedentes de El Dorado presentaron mayor porcentaje de colonización (figura 4B), probablemente se Citar como: Vallejos Torres, G., Saboya Pisco, A., \& Arevalo Lopez, L. A. (2021). Efecto Bioprotector de Micorrizas Arbusculares en la Reducción de Roya (Hemileia vastatrix) en la Región San Martín. Revista Agrotecnológica Amazónica, 1(1), 34-44. https://doi.org/10.51252/raa.v111.122 
adaptaron mejor a condiciones controladas. Estos resultados demuestran que las esporas de HMA lograron colonizar las raíces de las plantas de café, influenciando de manera bioprotectora y biofertilizante a cada planta, demostrando el gran efecto benéfico de estos microorganismos hacia el cultivo, además hubo resultados distintos entre tratamientos lo que podría estar influenciado por la presencia de consorcios micorrízicos propios de cada fuente de inóculo. La incidencia y severidad a roya se reduce cuando crece la longitud de micelio y colonización de micorrizas arbusculares tal como lo indica Herrera et al. (2019) quien evaluó la colonización micorrízica en muestras de suelos de plantas de café con y sin infección a roya. Otros investigadores han demostrado la efectividad de los hongos micorrízicos arbusculares contra muchas enfermedades de las plantas (Al-Ascar y Rashad, 2010; El-Sharkawy, 2010). Aunque los mecanismos no se entienden muy bien, laos HMA puede afectar la resistencia a las enfermedades de la planta huésped y mejorar la nutrición de la planta huésped y la salud posterior (Smith y Smith, 2011; Cavagnaro, 2014; Bowles et al. 2017a)

Los resultados reflejados en esta variable también tienen cierta similitud con lo encontrado por (Carbajal, 2009) quien también obtuvo una buena respuesta en porcentaje de colonización (43,3 $\%)$ por parte de los HMA nativos. De manera similar presenta (Del águila, 2016) donde el porcentaje de colonización de las plantas inoculadas con HMA-N en los diferentes tratamientos fluctuó entre 13,83\% y $30 \%$. (Escalona, 2002) encontró resultados similares en café con valores de porcentaje de colonización en vivero que fluctuaron de 23,6 \% a 42,96\% en plantas inoculadas con HMA. Así mismo (Rivera et al. 2003) reportó resultados entre $21 \%$ y 54 \% de colonización de HMA también en plantas de cafeto en la etapa de vivero. Estos resultados demuestran que las esporas de HMA al igual que en los resultados de longitud de micelio extraradical (MER), lograron colonizar las raíces de las plantas de café, influenciando de manera bioprotectora y biofertilizante a cada planta, además se demuestra que las esporas de HMA no se vieron perjudicadas por la presencia de roya, aparte se obtuvo resultados distintos entre tratamiento y tratamiento lo que podría estar influenciado por la presencia de consorcios Micorrízicos propios de cada fuente de inóculo. Por lo tanto, este estudio demuestra que la inoculación de plantas de café con hongos micorrízicos arbusculares autóctonos antes del establecimiento en el campo promueve el crecimiento de la planta de café (Vallejos-Torres et al. 2020)

\section{Conclusiones}

Las plantas de café variedad caturra inoculadas con HMA han mostrado mejor desarrollo en el aspecto morfológico y biométrico.

Las plantas inoculadas con HMA han demostrado mayor resistencia a la infección de la roya por que los resultados muestrean menor incidencia y severidad de la enfermedad.

Citar como: Vallejos Torres, G., Saboya Pisco, A., \& Arevalo Lopez, L. A. (2021). Efecto Bioprotector de Micorrizas Arbusculares en la Reducción de Roya (Hemileia vastatrix) en la Región San Martín. Revista Agrotecnológica Amazónica, 1(1), 34-44. https://doi.org/10.51252/raa.v1i1.122 


\section{Referencias bibliográficas}

Al-Ascar, A.A. y Rashad, Y.M. (2010). Arbuscular mycorrrhizal fungi: a biocontrol agent against common vean Fusarium root rot disease, Plant Pathol, J. 9,31-38.

Auge, R. M. 2001. Water relations, drought and vesicular-arbuscular mycorrhizal symbiosis. Mycorrhiza 11(1): 3-42.

Barrer, S. (2009). El uso de Hongos Micorrízicos Arbusculares como una alternativa para la agricultura. Universidad Industrial de Santander (UIS). Bucaramanga, Colombia. 10 pp.

Bock R. (2010). The give-and-take of DNA: horizontal gene transfer in plants. Trends Plant Sci. 15, 11-22. 10.1016/j.tplants.2009.10.001.

Bowles, T.M., Jackson, L.E. y Cavagnaro, T.R. (2017a). Los hongos micorrízicos mejoran la adquisición de nutrientes de las plantas y modulan la pérdida de nitrógeno con regímenes de agua variables. Glob. Cambiar Biol. 24, 171-182. doi: 10.1111 / gcb.13884.

Carbajal, E. (2009). Colonización Micorrízica por Hongos Vesículo Arbusculares en Hypericum, y Control del Nematodo Nodulador Meloidogyne Incognita. Escuela Politécnica del Ejército. Sangolquí, Ecuador. 148 pp.

Cavagnaro, T.R. (2014). Impactos de la aplicación de compost en la formación y funcionamiento de micorrizas arbusculares. Soil Biol. Biochem. 78, 38-44. doi: $10.1016 /$ j.soilbio.2014.07.007.

Chinchay, O. (2016). Efecto de Hongos Micorrízicos Arbusculares nativos sobre el nematodo agallador de raíces (Meloidogyne spp.) en plantones de café (Coffea arabica) variedad caturra en la Región San Martín-Universidad Nacional de San Martín - Tarapoto

Dalmazzone, C., Perrings y Williamson. (2002). Enfermedades y plagas exóticas: una perspectiva económica. Ekonomiaz No. 49, $1^{\circ}$ Cuatrimestre. Pp. 78-95

Del águila. K. (2016). Efecto de Hongos Micorrízicos Arbusculares nativos sobre el nematodo agallador de raíces (Meloidogyne spp.) en plantones de café (Coffea arabica) variedad caturra en la Región San Martín-Universidad Nacional de San Martín - Tarapoto- Perú

Diz, R. (2008). Métodos para evaluar normalidad y homogeneidad de varianza. Relación con el tamaño de muestra. Universidad de Granma. Bayamo. Cuba. 44p.

Escalona, P. (2002). Evaluación y caracterización de micorrizas arbusculares asociadas a Manihot sculenta sp. (Yuca) en dos regiones de la amazonía colombiana. Tesis Mag. Sc. Pontificia Universidad Javeriana. Bogotá, Colombia. 125 p.

El-Sharkawy, H.H.A. (2010). Control of Wilt Disease of Tomato through Application Natural Compost and Endomicorrhizal Fungy, PH.D.Thesis Faculty of Agriculture, Mansoura University, Egypt.

Espinosa Ruíz, G. C., and Vallejos Treminio, F. L. (2016).Desarrollo de formulaciones bioplaguicidas a base de Bauveria bassiana (Bals \& Vuils) con materiales sólidos y

Citar como: Vallejos Torres, G., Saboya Pisco, A., \& Arevalo Lopez, L. A. (2021). Efecto Bioprotector de Micorrizas Arbusculares en la Reducción de Roya (Hemileia vastatrix) en la Región San Martín. Revista Agrotecnológica Amazónica, 1(1), 34-44. https://doi.org/10.51252/raa.v1i1.122 
líquidos (Doctoral dissertation, Universidad Nacional Agraria) Managua, Nicaragua disponible en Página Web http://repositorio.una.edu.ni/id/eprint/3387

Fernández, C., Guadarrama, C., Sánchez, G. y Ramos, Z. (2005). Micorriza Arbuscular: Colonización intraradical y extracción de esporas del suelo. Tlaxcala. México.

Font, H. (2007). Estudio de precisión en la variable producción de huevos en gallinas White Leghorn. Tesis al título de Máster en Producción Animal. 84 p.

French, E. y Hebert, T. (1982). Métodos de investigación fitopatológica. Instituto Interamericano de Cooperación para la Agricultura. San José, Costa Rica.

Herrera, S., Castro, R., Moreno, J.P., Valdés, E. (2019). Diversidad endomicorrícica en plantas de café (Coffea arabica L.) infestadas con roya (Hemileia vastatrix). Nova scientia, 11(22), 102-123. https://doi.org/10.21640/ns.v11i22.1642

Ministerio de Agricultura y Riegos, MINAGRI. (2020). Reporte de precio y mercado de cacao. Perú

Herrera, S., Castro, R., Moreno, J.P. y Valdés, E. (2019). Diversidad endomicorrícica en plantas de café (Coffea arabica L.) infestadas con roya (Hemileia vastatrix). Nova scientia, 11(22), 102-123. https://doi.org/10.21640/ns.v11i22.1642

Padrón, E. (1996). Diseños experimentales con aplicación a la agricultura y la ganadería. 1 a Ed. Trillas. México. 215 p.

Palomino, A. C., López B. C., Espejo, J.R., Mansilla, S. R. y Quispe, V. J. (2014). Evaluación de la diversidad genética del café (Coffea arabica L.) en Villa Rica (Perú). Ecol. apl. 13(2): 129-134. http://www.scielo.org.pe/pdf/ecol/v13n2/a07v13n2.pdf

Rivera, R., Fernández, J., Hernández y Martín, T. (2003). El manejo eficiente de la simbiosis micorrízica, una vía hacia la agricultura sostenible. Estudio de caso: El Caribe. INCA.

Robles, C. (2009). "Variación temporal de la diversidad de hongos de micorriza arbuscular y el potencial micorrízico en especies silvestres de Agave en Oaxaca”. Instituto Politecnico Nacional (IPN). Oaxaca, México. 80 pp.

Sevilla, H. (2004). Recursos genéticos vegetales. Primera edición. Edit.Torre Azul SAC. Lima, Perú. 445 p.

Smith, S.E. y Smith, F.A. (2011). Roles of arbuscular mycorrhizas in plant nutrition and growth: new paradigms from cellular to ecosystem scales. Ann. Rev. Plant Biol. 62, 227-250.

Vallejos-Torres, G., Espinoza, E., Marín-Díaz, J. et al. The Role of Arbuscular Mycorrhizal Fungi Against Root-Knot Nematode Infections in Coffee Plants. J Soil Sci Plant Nutr (2020). https://doi.org/10.1007/s42729-020-00366-Z

Vierheilig, H., Coughlan, A.P, Wyss, U. y Piché, Y. (1998). La tinta y el vinagre, una técnica de tinción simple para los hongos micorrícicos arbusculares-. Applied and Environmental Microbiology, 64 (12), desde 5004 hasta 5007

Citar como: Vallejos Torres, G., Saboya Pisco, A., \& Arevalo Lopez, L. A. (2021). Efecto Bioprotector de Micorrizas Arbusculares en la Reducción de Roya (Hemileia vastatrix) en la Región San Martín. Revista Agrotecnológica Amazónica, 1(1), 34-44. https://doi.org/10.51252/raa.v1i1.122 


\section{Agradecimientos}

Un agradecimiento al Instituto de Investigaciones de la Amazonía Peruana.

\section{Anexos}

\section{Anexo 1:}

Cámara de conteo y rayado de Neubauer
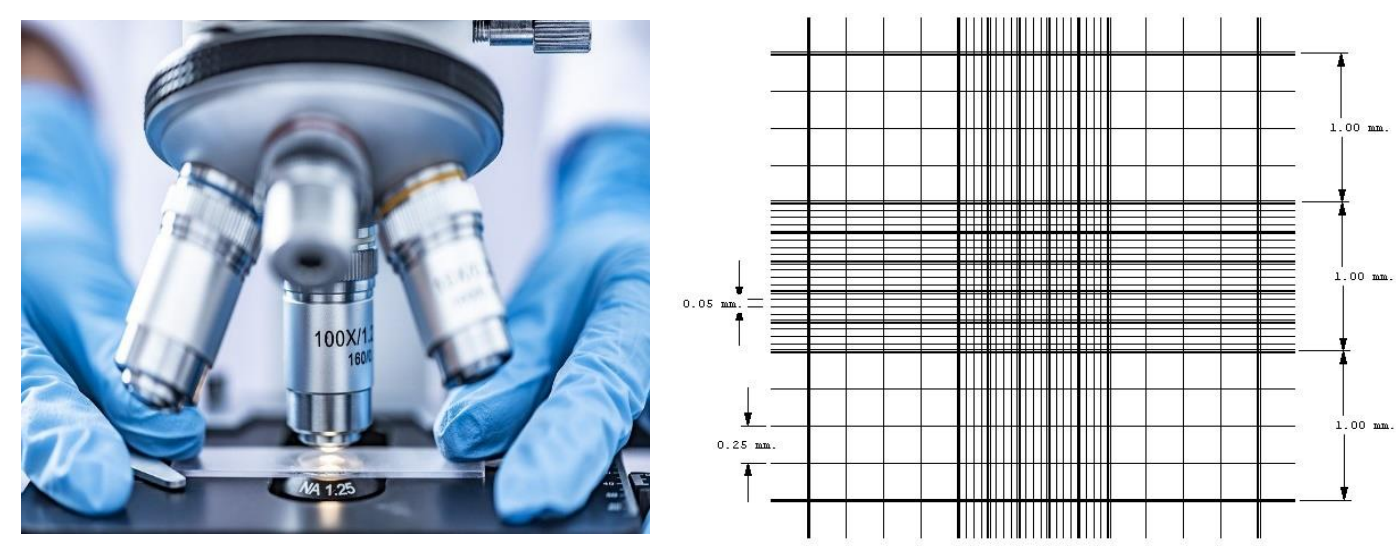

Cámara de conteo y rayado de Neubauer descrita por French y Hebert (1982), adaptado de Espinosa Ruíz, G. C. y Vallejos Treminio, F. L. (2016). 
Vol. 1, Núm. 1, ene-jun, 2021

\section{Conflicto de intereses}

Los autores consideran que no existen conflictos de interés para el presente estudio.

\section{Contribuciones de los autores}

Luis A. Arévalo López: seguimiento y apoyo al trabajo de investigación.

Anabel Saboya: Desarrollo de actividades y ensayo de investigación.

Geomar Vallejos-Torres: Asesoramiento y orientaciones al presente trabajo de investigación.

Citar como: Vallejos Torres, G., Saboya Pisco, A., \& Arevalo Lopez, L. A. (2021). Efecto Bioprotector de Micorrizas Arbusculares en la Reducción de Roya (Hemileia vastatrix) en la Región San Martín. Revista Agrotecnológica Amazónica, 1(1), 34-44. https://doi.org/10.51252/raa.v1i1.122 